\title{
Es tut mir leid, dass du nicht bleiben konntest
}

\author{
Alyson Gross \\ Medizinstudentin, Universität Zürich
}

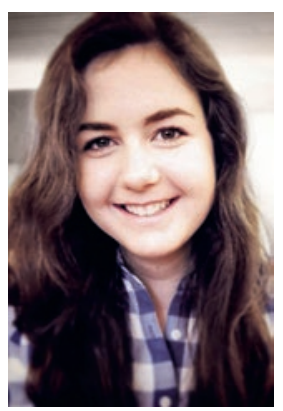

«Ich wusste nicht, was mich erwartete, als ich die antiken, moosigen Treppen zum Heiligengrab hochgeklettert war. Es war ein bedeckter Tag und niemand sonst besuchte den Tempel. Die Gärten waren in voller Blüte und schimmerten ruhig im trüben Licht. Als ich die oberste Stufe erreicht hatte, überwältigte mich der Anblick hunderter Jizo-Statuen auf so engem Raum. Die Feststellung, dass jede einzelne davon auf eine Familie mit ähnlichem Schicksal zurückging, machte mich traurig und wütend zugleich. Wir hatten diesen Ort ganz für uns und ich nahm mir genügend Zeit, um dessen Innenleben in mich aufzunehmen. Ich zündete eine Kerze an und erlaubte mir, meinen Kummer zu spüren.»

Joan wurde viermal schwanger, aber nie Mutter.

Vor einiger Zeit traf ich auf einen Satz von Vincent van Gogh in einem Brief an seinen Bruder Theo, der etwas in mir auslöste: «Manch einer hat ein grosses Feuer in seiner Seele, und nie kommt jemand, um sich daran zu wärmen. Die Vorbeigehenden sehen nur ein wenig Rauch über dem Schornstein und gehen dann ihrer Wege.» Diese Zeilen verfolgten mich die nächsten Tage; an wie vielen Menschen gehen wir nichtssagend vorbei, welche stürmende Feuer in sich tragen? Die mit Leidenschaft erfüllt oder schmerzverzehrt ihrer Wege gehen? Joan hatte ein solches Feuer in sich. Reproduktive Medikamente wie Menogon oder Clomifen funktionierten nicht; weder taten es zeitlich abgestimmter Beischlaf, zwei Operationen, zwei intra-uterine Inseminationen noch drei Runden IVF (In-vitro-Fertilisation). Das Ersparte wurde geplündert, denn die Hoffnung überwiegte. Kaffee, Jogging und überfüllte Arbeitswochen waren Dinge der Vergangenheit; Akupunktur, Vitamine und tägliche Injektionen gehörten neu zur gelebten Normalität.

Feuer kann ernsthaften Schaden hinterlassen. Anstatt die Wahrheit zu bekommen, wurde Joan einer Fruchtbarkeits-Fantasie ausgesetzt; von Monat zu Monat schwebend, gefangen in wiederholten Zyklen aufgebauter Hoffnung und entmutigender Enttäuschung. Was der Arzt ihr nie erzählt hatte: Eine künstliche Befruchtung scheitert öfter, als dass sie in einer erfolgreichen Schwangerschaft endet. Vielleicht hatte er ihr das aus gutmütigen Gründen verschwiegen, um die Hoffnungen seiner Patientin zu wahren. Doch wahrschein-

lich war es auch eine indirekte Folge der Massenvermarktung. Joan war damals 41 Jahre alt und ihre Chancen, auf natürliche Art schwanger zu werden, waren praktisch inexistent. Sie probierte es somit ein allerletztes Mal mithilfe einer Eizellspende.

Ich bin gleichermassen schockiert wie fasziniert von Joan und ihrer Geschichte. Es ist besonders erdrückend, auf die Zuneigung anderer angewiesen zu sein und gleichzeitig mit niemandem darüber sprechen zu können. Wir alle haben vielleicht selbst einmal in ferner Erinnerung von wiederkehrenden Fehlgeburten gehört, aber glaubten niemanden zu kennen, der so etwas selbst durchgemacht hatte. Dabei ist Infertilität häufiger als die Grippe.

Neue Reproduktionstechnologien können solche natürlichen Schranken überwinden. Damit bedeuten sie einerseits einen Zugewinn an Optionen, andererseits aber auch einen Zugewinn an Entscheidungsbelastung. Die Medikalisierung der Schwangerschaft könnte Frauen dazu drängen, Verfahren und Behandlungen vorzunehmen, welche nicht unbedingt im besten Sinne der Patientin liegen. Ich denke hierbei an Joan, welche erfolglos jahrelang enorme psychische und finanzielle Belastungen auf sich genommen hatte. Dabei lautet doch eines der frühsten medizinethischen Prinzipien «Primum non nocere». Hätten nicht in diesem Fall die am Patientenwohl orientierten Verpflichtungen den Arzt dazu veranlassen müssen, die Grenzen der Patientenautonomie wahrzunehmen?

Es gibt jedoch Werte im Leben, welche über medizinische oder klinische Vorteilsabwägungen hinausgehen. Dazu gehört meiner Meinung nach auch die reproduktive Freiheit einer Person. Nicht umsonst ist sie in der Schweiz als Grundrecht verankert und zusätzlich durch Menschenrechtskonventionen geschützt [1]. Mein Bauch gehört bekanntlich mir und für eine Frau ist es wichtig, entscheiden zu können, wann und wie sie Kinder kriegen möchte. Denn wir leben noch immer in einer Kultur, in welcher der Wert einer Frau, wenn auch unbewusst, von ihrer Zeugungsfähigkeit mitbestimmt wird:

«Und Gott segnete Noah und seine Söhne und sprach zu ihnen: Seid fruchtbar und mehret euch und füllet die Erde.» (Genesis 9:1) 
Bereits viele Jahrhunderte sind seit dieser Aufforderung Gottes vergangen, aber die Menschheit scheint diesem Gebot weiterhin mit Überschwang zu folgen. Assistierte Reproduktionstechnologien können damit eine Lösung für die Probleme vieler Frauen (und Männer) darstellen, aber gleichzeitig auch überholte Rollenbilder und Wertvorstellungen weiter verstärken. Joan selbst konnte sich lange nicht entscheiden, ob sie überhaupt ein Kind haben wollte. Doch je länger es dauerte schwanger zu werden, desto sicherer war sie sich, dass sie Mutter sein wollte. Ihr Kampf mit der Infertilität dauerte sieben Jahren an. Es war die grösste Herausforderung ihres Lebens, aber vielleicht genau die Erfahrung, die sie brauchte, um sich in ihrer Rolle als Mutter zu verfestigen. Adoption kam für sie und ihren Ehemann Paul jedenfalls nie in Frage. Dafür waren sie in ihrem Glauben an ein medizinisches Wunder zu optimistisch. Ihre grösste Sorge war lediglich, das richtige "Timing» der einzelnen Injektionen und Arztbesuche einzuhalten. Paul war dafür zuständig, die Medikamente und Injektionsspritzen zuhause vorzubereiten, während Joan sich diese dann selbst im eigenen Badezimmer zuführte. Und dies Tag für Tag.

Obwohl ich sie beide als liebevolles und starkes Paar kenne und weiss, dass sie in jeder Situation offen miteinander kommunizieren können, kann ich nicht glauben, dass sie jene Prozedur so lange freiwillig auf sich nahmen. Ich kann mich noch gut daran erinnern, wie sie mir Ultraschall-Videos von ihren Kindern zeigten, mir ihre Spitznamen verrieten und wie alt sie heute wären. Wie schafft es jemand, über den Verlust eines Kindes hinwegzukommen? Und wie fasst man den Mut, sich nochmals in die gleiche Situation zu begeben? Ich sehe heute noch Joan vor Augen, wie sie auf den Bildschirm starrt, kurz innehält und mit einem nachdenklichen Ton beteuert: Es tut mir leid, dass du nicht bleiben konntest.

Dennoch zeigt mir ihre Bereitschaft, tausende US-Dollar auszugeben und ihre eigene Gesundheit zu riskieren, dass es ein starkes emotionales Begehren nach biologischer Nachkommenschaft gibt. Unfruchtbarkeit ist ein Trauma, auch wenn es nicht lebensbedrohlich ist. Ob Joan in ihrer Vorgehensweise moralisch richtig lag, weiss ich nicht. Doch schwierige Entscheidungen können nicht mithilfe eines strengen Paternalismus getroffen werden. Sie müssen stattdessen anhand aufklärerischen Einübens ausreifen. Wie Simone Veil bereits andeutete: "C'est toujours un drame», und mir scheint die Möglichkeit zu entscheiden wichtiger als die Entscheidung selbst.

"Unsere Reise nach Japan war ein bewusster Versuch, mit vergangenen Enttäuschungen abzuschliessen. Bevor wir uns auf unsere Heimfahrt begeben und wieder versuchen würden, eine Familie zu gründen, kehrte ich zum Tempel zurück. Es war ein sonniger Nachmittag und riesige Touristengruppen ergossen sich aus überfüllten Bussen. Die Gärten waren noch immer schön, aber diesmal strahlend und bewegt. Ich kletterte wieder die Treppen hinauf und spürte, dass sich die Schwere des Ortes aufgelöst hatte. Die Verwandlung, auf die ich gehofft, aber nicht erwartet hatte, war eingetreten. Allerdings blieb der Entscheid "aufzugeben» unmöglich. Meine Chancen würden zwar immer geringer, doch das kleine Stück Hoffnung verführte mich.»

- Joan wurde zum fünften Mal schwanger, und endlich Mutter.

Literatur

Siehe Art. 14 BV; Art. 12 EMRK. 